\title{
Stuart L. Houser, M.D.: The Operated Heart at Autopsy
}

\author{
Humana Press, Totowa, 2009, Price EUR 119.95, ISBN 978-1-60327-807-2
}

\author{
Claas T. Buschmann
}

Accepted: 2 October 2009/Published online: 27 October 2009

(C) Humana Press 2009

Autopsies in cases of cardiac-failure fatalities which have undergone previous surgical treatment in the recent or remote past may address unique anatomical, pathophysiological, technical and forensic challenges to the forensic pathologist needing to understand the pathways leading to the patient's demise. When a past medical history is missing, what is the appropriate way of determining what surgical treatment was done? Was the cause of death heartrelated? What was the impact of heart surgery on the cause of death? Which steps should be taken during autopsy, and in what order? What is the importance of technical issues of the heart surgery in a given autopsy? These and other questions can be adequately answered by "The Operated Heart at Autopsy”, a monograph by Stuart L. Houser, M.D.

Dr. Houser has a background in the field of cardiac surgery (17 years experience) as well as in the field of cardiac pathology (12 years experience). Thus, having an interdisciplinary approach, the author takes the reader through common and uncommon cardiac surgery procedures as well as inborn and acquired infant and adolescent cardiac diseases, pronouncing the significance and role of heart operations during autopsy in cases of suspected heartrelated deaths and describing autopsy techniques step by step.

The books consists of well-explained chapters on e.g. congenital heart diseases and surgical footprints, external evidence of open-heart-surgery, and technical issues in cardiac surgery such as uni- and biventricular assist devices, intraaortic balloon pump, and coronary artery bypass grafting and other surgical techniques. It also addresses some problems and answers a lot of questions on the priorities of cardiac surgery and forensic pathology, as well as presentation and discussion of 20 interesting and well-documented autopsy cases, practical instructions and suggestions, e.g. execution of detaching the cardiopulmonary block, dissection of the operated heart and techniques of post-mortem coronary injection are presented. The references are plentiful, up-to-date and allow further gathering of information.

The book also contains 91 colour photographs with comments of autopsy findings and technical issues. Nevertheless, even more high-quality pictures and some schematic drawings, explaining surgical procedures and making the (non-cardiac-surgeon) reader understand the purpose of some heart operations might be added in a following edition as well as a short chapter on cardiac surgery following traumatic incidents.

As this book not only addresses the need of pathologists in practice and training to achieve a subsequent level of competence in dealing with the anatomic complexity of operated hearts, but also is a felicitous example of amplified interlocking between forensic and clinical disciplines (which ought to be strived for in the future), it should be included in the library of any pathology department and autopsy pathologist in charge of suspected fatalities related to cardiac surgery.
C. T. Buschmann $(\bowtie)$

Institute of Legal Medicine and Forensic Sciences, University

Medical Centre Charité, Turmstr. 21, Building L, 10559 Berlin,

Germany

e-mail: claas.buschmann@charite.de 